\title{
Synthesis and antimicrobial activity of novel $(3 \mathrm{a}, S)-1-($ aminoacid ester)-3a,4-dihydro-3H-1 $\lambda^{5}-[1,3,2]$ oxazaphospholo $[3,4-a]$ indol-1-oxides
}

\author{
A.Uma Ravi Sankar, B. Siva Kumar, M. Veera Narayana Reddy, B. Haribabu, \\ and C. Naga Raju* \\ Department of Chemistry, Sri Venkateswara University, Tirupati-517502, India \\ E-mail: naga raju04@yahoo.co.in
}

\begin{abstract}
Synthesis of $(3 \mathrm{a}, S)-1-\left(\right.$ amino acid ester)-3a,4-dihydro-3H-1 $\lambda^{5}-[1,3,2]$ oxazaphospholo[3, 4$a$ ]indol-1-oxides was accomplished through a two-step process involving preparation of the monochloride and its subsequent reaction with amino acid ester hydrochlorides in dry tetrahydrofuran in the presence of triethylamine at various temperatures. All the title compounds were characterized by IR, ${ }^{1} \mathrm{H},{ }^{13} \mathrm{C},{ }^{31} \mathrm{P} \mathrm{NMR}$ and mass spectral data. They exhibited significant antimicrobial activity.
\end{abstract}

Keywords: (2S)-2,3-Dihydro-1H-2-indolyl methanol, amino acid esters, triethylamine, (3a,S)-1-chloro-3a, 4-dihydro-3H-1 $\lambda^{5}-[1,3,2] \quad$ oxaazaphospholo[3,4-a]indol-1-oxides, antimicrobial activity

\section{Introduction}

Phosphoramides substituted with an amino acid ester are an important class of rationally designed therapeutics for antineoplastic properties. ${ }^{1-5}$ The attachment of an amino acid group to the phosphate moiety is expected to increase cellular uptake and thus enhance chemotherapeutic properties. Organophosphorus compounds are used as insecticides, agricultural and horticultural pesticides and veterinary medicines. They are also used in human medicines and in various public hygiene products for use both by professional operators and the general public. Pesticides in soil have far reaching consequences as they disturb the delicate equilibrium between microorganisms and their environment. ${ }^{6}$ Bioremediation is a promising area which holds potential for eco-restoration of pesticide contaminated soil. Several microorganisms are able to degrade a large variety of compounds. ${ }^{7}$

The presence of an exocyclic P-N bond in an amino acid ester attached to a benzoxazaphosphorin system is expected to increase cellular uptake of their chemotherapeutic 
properties. Further, hydrolysis of these novel heterocycles may release products of limited toxicity to the system. In view of this, we have synthesized new heterocycles in which amino acid esters are linked to a phosphorus atom, studied their antimicrobial activity and phosphate degradation potential in several bacterial species.

\section{Results and Discussion}

Synthesis of the compounds (5a-h) was accomplished through two steps. Reaction of (2S)-2,3dihydro-1H-indol-2-ylmethanol (1) with phosphorus oxychloride in equimolar quantities in the presence of triethylamine in dry tetrahydrofuran at $10-30{ }^{\circ} \mathrm{C}$ produced the corresponding monochoride, $(3 \mathrm{a}, S)$-1-chloro-3a,4-dihydro-3H-1$\lambda^{5}$-[1,3,2]oxaza- phospholo[3,4- $a$ ]indol-1-oxide (3). This was subsequently reacted with various amino acid ester hydrochlorides, in dry tetrahydrofuran in the presence of triethylamine to obtain the title compounds in moderate yields $(52-69 \%)$.

The second step of the reaction was run at $20-40{ }^{\circ} \mathrm{C}$ with stirring for $6-8 \mathrm{~h}$, with progress monitored by TLC. In all the reactions, the crude title compounds were separated by removing the solvent from the filtrate in a rota-evaporator. They were purified by flash chromatography using hexane-ethyl acetate $(6: 2)$ step gradient mixtures as eluents and characterized by elemental analysis, IR, ${ }^{1} \mathrm{H},{ }^{13} \mathrm{C}$ and ${ }^{31} \mathrm{P} \mathrm{NMR}$ and mass spectral data. ${ }^{8}$

Compounds 5a-h showed absorption bands in the region 1215-1282 $\mathrm{cm}^{-1}$ for $(\mathrm{P}=\mathrm{O})$ and $3446-3408 \mathrm{~cm}^{-1}$ for $(\mathrm{P}-\mathrm{NH})$ confirming the presence of phosphoryl $(\mathrm{P}=\mathrm{O})$ and $\mathrm{P}-\mathrm{NH}$ functional groups in these compound. ${ }^{12}$ The aromatic protons of $\mathbf{5 a - h}$ resonated as multiplets at $\delta$ 7.676.94. The C-4 methylene protons resonated as multiplets at $\delta 4.46-4.02$ indicating their nonequivalence and coupling with phosphorus ${ }^{12,13}$ The ${ }^{13} \mathrm{C}$ NMR chemical shifts of 5a, $5 \mathbf{b}$, 5e and 5h were interpreted on the basis of additivity rules. The phosphorus bonded C-3 resonated as a singlet at $\delta$ 52.7-59.7.The chiral carbon (C-3a) gave a singlet in the region $\delta$ 45.4-45.6. The remaining carbon signals were observed in the expected region. ${ }^{8}$ The ${ }^{31} \mathrm{P}$ NMR spectra of $\mathbf{5 a - h}$ showed phosphorus resonance signals ${ }^{8,9}$ in the region 2.16 to $12.12 \mathrm{ppm}^{10}$ Thus, the combined analytical, IR and NMR data agreed conclusively with the proposed structures for $\mathbf{5 a}-\mathbf{h}$.

\section{Antimicrobial activity}

The compounds 5a-h were screened for their antibacterial activity against Staphylococcus aureus (gram +ve) and Escherichia coli (gram -ve) by the disc diffusion method ${ }^{12,13}$ in nutrient agar medium at three different concentrations $(25,50,100 \mathrm{~g} /$ disc) in dimethyl formamide (DMF). These solutions were added to each filter paper disc and DMF was used as control. The plates were incubated at $35{ }^{\circ} \mathrm{C}$ and examined for zone of inhibition around each disc after $24 \mathrm{~h}$. The results were compared with the activity of the standard antibiotic Streptomycin $(50 \mu \mathrm{g} / \mathrm{disc})$. Their antifungal activity was evaluated against Aspergillus niger and Helminthosporium oryzae at three different concentrations $(100,50,25 \mu \mathrm{g} /$ disc) and Bavestin was used as the reference compound. Fungal 
cultures were grown on potato dextrose broth at $25{ }^{\circ} \mathrm{C}$ and spore suspension was adjusted to $10^{5}$ spores/mL. Most of the compounds showed moderate activity against bacteria and low activity on fungi. Each test was done in triplicate and the mean of the diameter of the inhibition zones was calculated. Controls included the use of the solvent DMF without test compounds: no antibacterial activity was noticed for the solvent (DMF) employed in the test.

\section{Conclusions}

In summary, we have reported an effective and simple reaction for the synthesis of novel phosphorus heterocyclic compounds containing a chiral centre with amino acid moieties linked to phosphorus. The majority of the compounds (5a-h) exhibited moderate activity against bacteria and fungi when compared to that of the respective standards.

\section{Experimental Section}

General procedures. All melting points were determined in open capillary tubes on a Mel-temp apparatus and are uncorrected. Microanalysis was performed at the Central Drug Research Institute, Lucknow, India. Infrared spectra $\left(v_{\max }\right.$ in $\left.\mathrm{cm}^{-1}\right)$ were recorded as $\mathrm{KBr}$ pellets on a Perkin-Elmer 283 double beam spectrophotometer. ${ }^{1} \mathrm{H},{ }^{13} \mathrm{C}$ and ${ }^{31} \mathrm{P}$ NMR spectra were recorded on AMX $400 \mathrm{MHz}$ spectrometer operating at $400 \mathrm{MHz}$ for ${ }^{1} \mathrm{H}, 100 \mathrm{MHz}$ for ${ }^{13} \mathrm{C}$, and $161.9 \mathrm{MHz}$ for ${ }^{31} \mathrm{P}$, using deuterochloroform as solvent. The ${ }^{1} \mathrm{H}$ and ${ }^{13} \mathrm{C}$ NMR chemical shifts were referenced to tetramethylsilane, and ${ }^{31} \mathrm{P}$ chemical shifts to $85 \% \mathrm{H}_{3} \mathrm{PO}_{4}$. Mass spectra were recorded on a Jeol SX $102 \mathrm{DA} / 600$ mass spectrometer using Argon/Xenon (6 KV, $10 \mathrm{~mA})$ as the FAB gas and also on a Shimadzu QP-2000 GC-MS instrument. Refractive indices were recorded using a SEPA-300 instrument and a $10 \mathrm{~cm}$ tube at $28^{\circ} \mathrm{C}$.

\section{(2S)-2, 3-Dihydro-1H-2-indolyl methanol (1)}

To a stirred and ice-cold suspension of $\mathrm{LiAlH}_{4}(3.10 \mathrm{~g}, 0.0817 \mathrm{~mol})$ in dry THF $(100 \mathrm{~mL})$, a solution of ethyl $2 S$-indole-2-carboxylate $(7.60 \mathrm{~g}, 0.039 \mathrm{~mole})$ in dry THF $(35 \mathrm{~mL})$ was added dropwise. The mixture was stirred and refluxed for $3 \mathrm{~h}$ and then cooled, and the excess of $\mathrm{LiAlH}_{4}$ was decomposed by the addition of a mixture of water $(6.2 \mathrm{~mL})$ and THF $(50 \mathrm{~mL})$. After stirring at room temperature for $1 \mathrm{~h}$, the inorganic salts were filtered off and washed with hot ethyl acetate $(150 \mathrm{~mL})$. The combined organic filtrate and washings were dried $\left(\mathrm{Na}_{2} \mathrm{SO}_{4}\right)$ and evaporated under reduced pressure to give the crude amino alcohol ${ }^{14}$ as a crystalline product, which was filtered off, washed with $\mathrm{Et}_{2} \mathrm{O}$ and recrystallised from EtOAc, yield: $4.03 \mathrm{~g}(68 \%)$, m.p. $127^{\circ} \mathrm{C}$. 


\section{Proline ethyl ester hydrochloride (3a)}

Thionyl chloride $(0.7 \mathrm{~mL}, 0.01 \mathrm{~mol})$ was added slowly to methanol $(25 \mathrm{~mL})$ at $0{ }^{\circ} \mathrm{C}$ then proline $(1.31 \mathrm{~g}, 0.01 \mathrm{~mol})$ was dissolved in it. The solution was refluxed for 4 hours. Solvent was removed in a rota-evaporator, the crude proline methyl ester hydrochloride, was triturated with ether at $0{ }^{\circ} \mathrm{C}$ until excess dimethyl sulfite was removed. The resulting solid product was collected and dried under vacuum, to give crude methyl ester hydrochloride. The crude material was dissolved in minimum amount of hot methanol. Slow addition of excess of ether followed by cooling to $0{ }^{\circ} \mathrm{C}$ gave pure crystals. They were washed twice with ether:methanol $(5: 1)$ and dried under vacuum to get proline methyl ester hydrochloride, ${ }^{15}$ yield $25 \mathrm{~g}(80 \%), \mathrm{mp} 148-150{ }^{\circ} \mathrm{C}$. Other amino acid esters were prepared using the above procedure.

(3a,S)-1-Chloro-3a,4-dihydro-3H-1 $\lambda^{5}-[1,3,2]$ oxazaphospholo[3, 4-a]indol-1-oxide (3) A solution of phosphorus oxychloride $(0.02 \mathrm{~mol})$ in $10 \mathrm{~mL}$ of dry tetrahydrofuran was added dropwise to a stirred solution of (1) $(0.02 \mathrm{~mol})$ and triethylamine $(0.04 \mathrm{~mol})$ in $20 \mathrm{~mL}$ of dry tetrahydrofuran at $0{ }^{\circ} \mathrm{C}$ over a period of $30 \mathrm{~min}$. After stirring for $6 \mathrm{~h}$ at $10-30{ }^{\circ} \mathrm{C}$, formation of

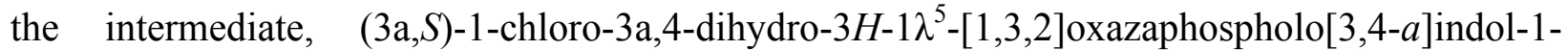
oxide (3) was ascertained by TLC analysis. Triethylamine hydrochloride was separated by filtration and the reaction mixture was used for the next reaction step without further purification.

Typical Procedure. (3a,S)-1-(Glycine ethyl ester)-3a,4-dihydro-3H-1 $\lambda^{5}-[1, \quad 3, \quad 2]$ oxazaphospholo[3,4-a]indol-1-oxide (5a)

Glycine ethyl ester $(0.02 \mathrm{~mol})$ in $10 \mathrm{~mL}$ of tetrahydrofuran was added to the reaction mixture of 3 at $10{ }^{\circ} \mathrm{C}$ with stirring in the presence of triethylamine $(0.04 \mathrm{~mol})$. The reaction mixture was slowly raised to $40{ }^{\circ} \mathrm{C}$ and maintained at this temperature for $5 \mathrm{~h}$ with stirring. The progress of the reaction was monitored by TLC. After the completion of the reaction, triethylamine hydrochloride was separated by filtration. The solvent was evaporated under reduced pressure to obtain a solid residue, which was washed with water and recrystallised from methanol to yield the title compound (5a). Compounds $\mathbf{5} \mathbf{b}-\mathbf{h}$ were prepared by the above procedure.

\section{Determination of minimum inhibitory concentration (MIC)}

Minimum inhibitory concentration (MIC) for the compounds 5a-h. Compounds 5a-h concentrations of $0.1-5.6 \mu \mathrm{g} / \mathrm{mL}$ in steps of $100 \mu \mathrm{g} / \mathrm{mL}$ were evaluated. Specifically $0.1 \mathrm{~mL}$ of standardized inoculum $\left(1.2 \times 10^{7} \mathrm{CFU} / \mathrm{mL}\right)$ was added to each tube. The tubes were incubated aerobically at $37{ }^{\circ} \mathrm{C}$ for $18-24 \mathrm{~h}$. Two control tubes were maintained for each test batch. These included antibiotic control (tube containing compounds $\mathbf{5 a - h}$ and the growth medium without inoculum) and organism control (the tube containing and growth medium, physiological saline and the inoculum). The lowest concentration (highest dilution) of the compounds 5a-h that produced no visible bacterial growth (no turbidity) when compared with the control tubes was regarded as MIC ${ }^{16}$ (Table 2). 

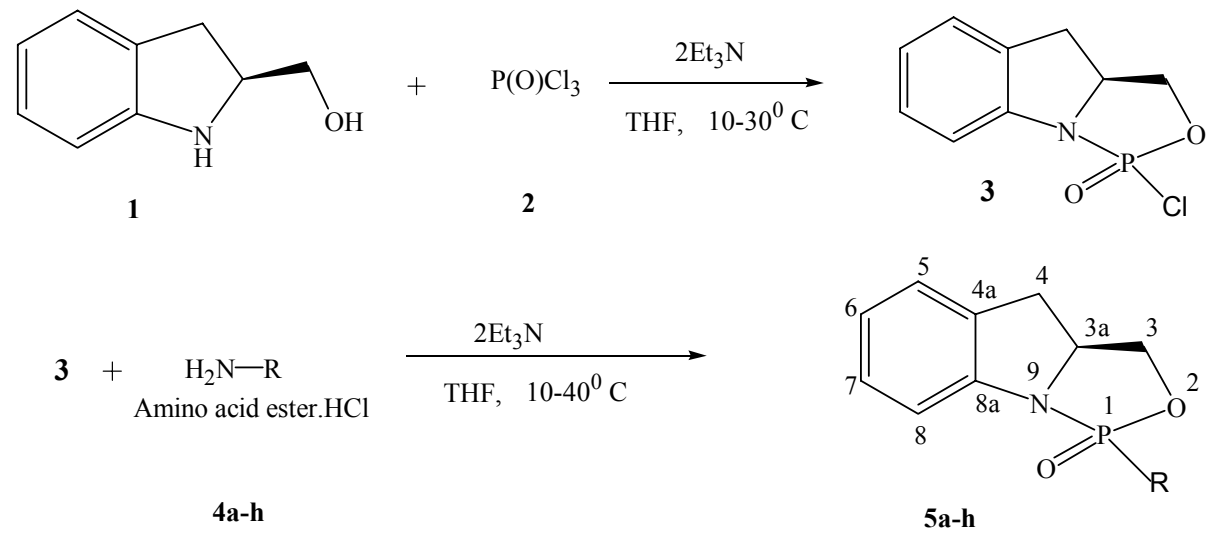

$\mathrm{R}$
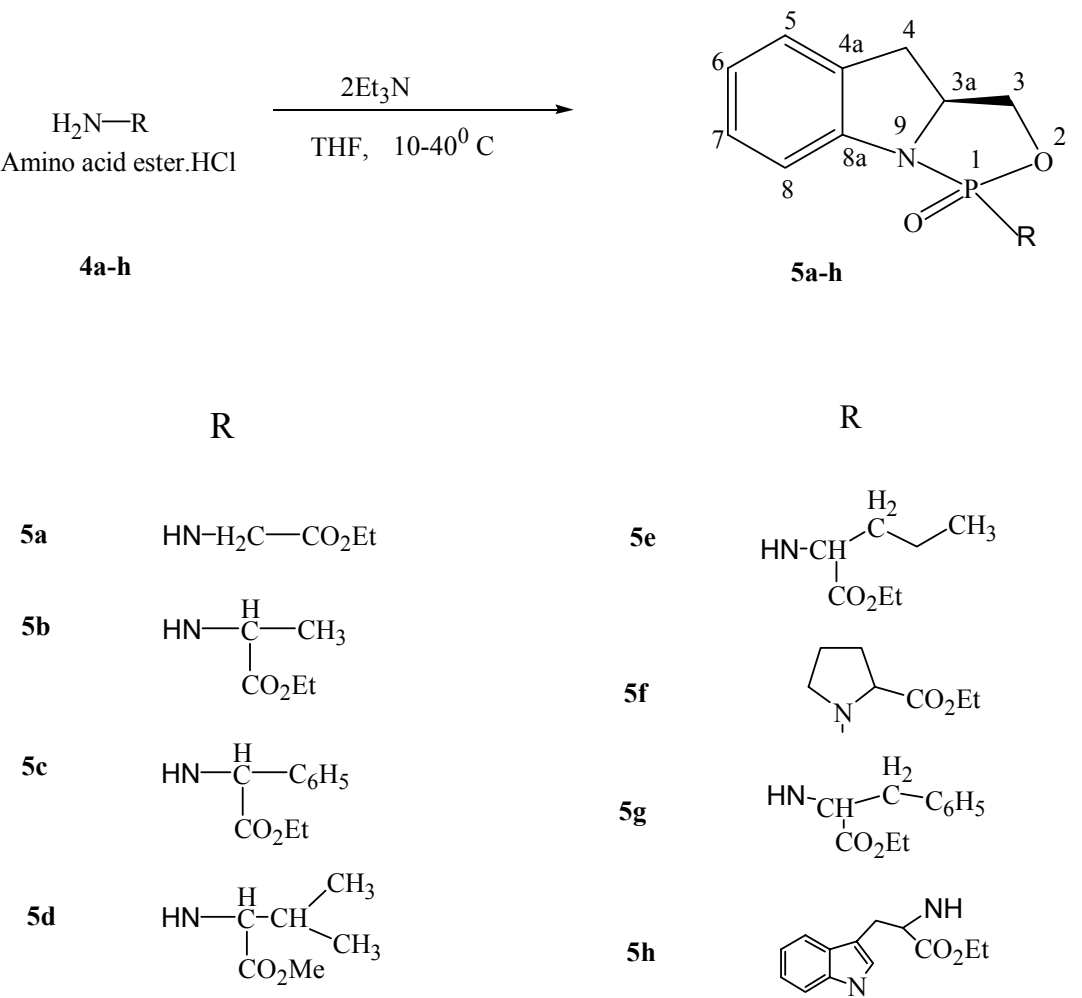

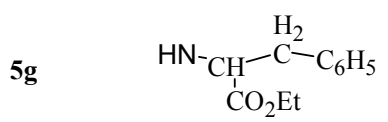

$5 \mathbf{h}$

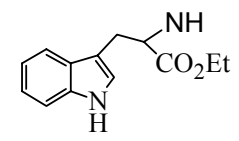

\section{Scheme 1}

Physical, analytical and spectral data for the 5a-h

(3a,S)-1-(Glycine ethyl ester)-3a,4-dihydro-3H-1 $\lambda^{5}-[1,3,2]$ oxazaphospholo[3,4-a] indol-1oxide (5a). Yield 60\%, m.p. 120-122 ${ }^{\circ} \mathrm{C}$. IR (KBr): $v_{\max } 3412(\mathrm{NH}), 1258(\mathrm{P}=\mathrm{O}), 1746(\mathrm{C}=\mathrm{O})$ $\mathrm{cm}^{-1} ;{ }^{31} \mathrm{P} \mathrm{NMR}\left(\mathrm{CDCl}_{3}, 161.9 \mathrm{MHz}\right) \delta: 6.06,{ }^{1} \mathrm{H} \mathrm{NMR}\left(\mathrm{CDCl}_{3}, 400 \mathrm{MHz}\right) \delta: 7.02-7.48(\mathrm{~m}, 4 \mathrm{H}$, Ar-H), 3.08 (d, $\left.J=6.5 \mathrm{~Hz}, 2 \mathrm{H}, \mathrm{CH}_{2}\right), 4.16-4.27$ (m, 1H, CH), 9.84 (br s , 1H, P-N-H), 3.78 (d, $J$ $\left.=31.4 \mathrm{~Hz}, 2 \mathrm{H},-\mathrm{OCH}_{2}-\right), 3.65\left(\mathrm{q}, 2 \mathrm{H}, \mathrm{OCH}_{2} \mathrm{CH}_{3}\right), 2.36\left(\mathrm{~s}, 2 \mathrm{H}, \mathrm{CH}_{2}\right), 1.21\left(\mathrm{t}, 3 \mathrm{H}, \mathrm{OCH}_{2} \mathrm{CH}_{3}\right),{ }^{13} \mathrm{C}$

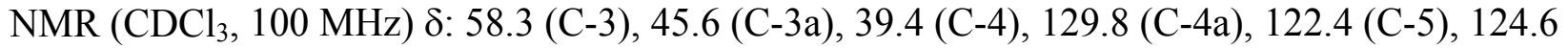
(C-6), 113.7 (C-7), 128.9 (C-8), 138.4 (C-8a), $174.5(\mathrm{C}=\mathrm{O}), 53.4\left(\mathrm{CH}_{2}\right), 49.8\left(\mathrm{OCH}_{2}\right), 17.9$ $\left(\mathrm{OCH}_{2} \mathrm{CH}_{3}\right)$; Anal. Calcd. for $\mathrm{C}_{13} \mathrm{H}_{17} \mathrm{~N}_{2} \mathrm{O}_{4} \mathrm{P}$ : C, 52.70; H, 5.78; N, 9.46. Found: C, 52.65; H, 5.74; N, 9.42\%, $[\alpha]_{\mathrm{D}}(+) 3.0$.

(3a,S)-1-(Alanine ethyl ester)-3a,4-dihydro-3H-1 $\lambda^{5}-[1,3,2]$ oxazaphospholo $[3,4-a]$ indol-1oxide (5b). Yield 65\%, m.p. $135-137^{\circ} \mathrm{C}$. IR (KBr): $v_{\max } 3418(\mathrm{NH}), 1246(\mathrm{P}=\mathrm{O}), 1754(\mathrm{C}=\mathrm{O})$ $\mathrm{cm}^{-1} ;{ }^{31} \mathrm{P} \mathrm{NMR}\left(\mathrm{CDCl}_{3}, 161.9 \mathrm{MHz}\right) \delta: 12.02,{ }^{1} \mathrm{H} \mathrm{NMR}\left(\mathrm{CDCl}_{3}, 400 \mathrm{MHz}\right) \delta: 7.09-7.58(\mathrm{~m}, 4 \mathrm{H}$, Ar-H), 3.03 (d, $\left.J=6.2 \mathrm{~Hz}, 2 \mathrm{H}, \mathrm{CH}_{2}\right), 4.06-4.14$ (m, 1H, CH), 9.88 (br s , 1H, P-N-H), 3.72 (d, $J$ 
$\left.=31.4 \mathrm{~Hz}, 2 \mathrm{H},-\mathrm{OCH}_{2}-\right), 3.48\left(\mathrm{q}, 2 \mathrm{H}, \mathrm{OC}_{2} \mathrm{CH}_{3}\right), 1.19\left(\mathrm{t}, 3 \mathrm{H}, \mathrm{OCH}_{2} \mathrm{CH}_{3}\right), 1.32\left(\mathrm{~s}, 3 \mathrm{H}, \mathrm{CH}_{3}\right)$;

${ }^{13} \mathrm{C} \mathrm{NMR}\left(\mathrm{CDCl}_{3}, 100 \mathrm{MHz}\right)$ 8: 59.4 (C-3), 45.4 (C-3a), 39.8 (C-4), 129.6 (C-4a), 122.9 (C-5), 124.4 (C-6), 113.8 (C-7), 129.7 (C-8), 136.4 (C-8a), $170.8(\mathrm{C}=\mathrm{O}), 53.6(\mathrm{CH}), 48.7\left(\mathrm{OCH}_{2}\right), 17.4$ $\left(\mathrm{OCH}_{2} \mathrm{CH}_{3}\right)$; Anal. Calcd. for $\mathrm{C}_{14} \mathrm{H}_{19} \mathrm{~N}_{2} \mathrm{O}_{4} \mathrm{P}: \mathrm{C}, 54.19 ; \mathrm{H}, 6.17$; N, 9.03\% Found: C, 54.13; H, $6.14 ; \mathrm{N}, 9.00 \%,[\alpha]_{\mathrm{D}}(+) 3.4$.

(3a,S)-1-(Phenyl glycine ethyl ester)-3a,4-dihydro-3H-1 ${ }^{5}-[1,3,2]$ oxazaphospholo- $[3,4-$ a]indol-1-oxide (5c). Yield 52\%, m.p. 147-149 ${ }^{\circ} \mathrm{C}$. IR (KBr): $v_{\max } 3446(\mathrm{NH}), 1242(\mathrm{P}=\mathrm{O}), 1744$ $(\mathrm{C}=\mathrm{O}) \mathrm{cm}^{-1} ;{ }^{31} \mathrm{P} \mathrm{NMR}\left(\mathrm{CDCl}_{3}, 161.9 \mathrm{MHz}\right) \delta: 2.16,{ }^{1} \mathrm{H} \mathrm{NMR}\left(\mathrm{CDCl}_{3}, 400 \mathrm{MHz}\right) \delta: 7.09-7.49$ (m, 9H, Ar-H), 3.03 (d, $J=6.4 \mathrm{~Hz}, 2 \mathrm{H}, \mathrm{CH}_{2}$ ), 4.02-4.09 (m, 1H, CH), 9.81 (br s , 1H, P-N-H), 3.87 $\left(\mathrm{d}, J=32.1 \mathrm{~Hz}, 2 \mathrm{H},-\mathrm{OCH}_{2}-\right), 4.48-4.56(\mathrm{~m}, 1 \mathrm{H}, \mathrm{CH}), 3.65$ (q, 2H, $\left.\mathrm{OC}_{2} \mathrm{CH}_{3}\right), 1.13$ ( t, 3H, $\left.\mathrm{OCH}_{2} \underline{\mathrm{C}}_{3}\right), 1.19$ (d, 2H, $-\mathrm{CH}_{2}$ ); Anal.Calcd. for $\mathrm{C}_{19} \mathrm{H}_{21} \mathrm{~N}_{2} \mathrm{O}_{4} \mathrm{P}: \mathrm{C}, 61.29 ; \mathrm{H}, 5.68 ; \mathrm{N}, 7.52 \%$ Found: C, 61.25; H, 5.64; N, 7.50\%, $[\alpha]_{\mathrm{D}}(+) 2.5$.

(3a,S)-1-(Valine methyl ester)-3a,4-dihydro-3H-1 $\lambda^{5}-[1,3,2]$ oxazaphospholo[3,4-a] indol-1oxide (5d). Yield 68\%, m.p. 172-175 ${ }^{\circ} \mathrm{C}$. IR (KBr): $v_{\max } 3435(\mathrm{NH}), 1282(\mathrm{P}=\mathrm{O}), 1752(\mathrm{C}=\mathrm{O})$ $\mathrm{cm}^{-1} ;{ }^{31} \mathrm{P} \mathrm{NMR}\left(\mathrm{CDCl}_{3}, 161.9 \mathrm{MHz}\right) \delta: 6.62,{ }^{1} \mathrm{H} \mathrm{NMR}\left(\mathrm{CDCl}_{3}, 400 \mathrm{MHz}\right) \delta: 7.08-7.67(\mathrm{~m}, 4 \mathrm{H}$, Ar-H), 3.22 (d, $\left.J=6.4 \mathrm{~Hz}, 2 \mathrm{H},-\mathrm{CH}_{2}-\right), 4.28-4.46(\mathrm{~m}, 1 \mathrm{H},-\mathrm{CH}), 9.78(\mathrm{~s}, 1 \mathrm{H}, \mathrm{P}-\mathrm{NH}), 3.75(\mathrm{~d}, J=$ $33.8 \mathrm{~Hz}, 2 \mathrm{H},-\mathrm{OCH}_{2}$ ) , 4.62-4.78 (m, 1H, -CH-), 3.54 (q, 2H, $-\mathrm{OCH}_{2}$ ), $1.17\left(\mathrm{t}, 3 \mathrm{H},-\mathrm{CH}_{3}\right)$; Anal.Calcd. for $\mathrm{C}_{15} \mathrm{H}_{2}{ }_{1} \mathrm{~N}_{2} \mathrm{O}_{4} \mathrm{P}: \mathrm{C}, 55.55 ; \mathrm{H}, 6.53 ; \mathrm{N}, 8.64 \%$ Found: C, 55.50; H, 6.48; N, 8.60 $\%,[\alpha]_{\mathrm{D}}(+) 2.9$.

(3a,S)-1-(nor-Valine ethyl ester)-3a,4-dihydro-3H-1 $\lambda^{5}-[1,3,2]$ oxazaphospholo[3,4-a] indol1-oxide (5e). Yield 67\%, m.p. 130-132 ${ }^{\circ} \mathrm{C}$. IR (KBr): $v_{\max } 3442(\mathrm{NH}), 1261(\mathrm{P}=\mathrm{O}), 1743(\mathrm{C}=\mathrm{O})$ $\mathrm{cm}^{-1} ;{ }^{31} \mathrm{P} \mathrm{NMR}\left(\mathrm{CDCl}_{3}, 161.9 \mathrm{MHz}\right) \delta: 7.96,{ }^{1} \mathrm{H} \mathrm{NMR}\left(\mathrm{CDCl}_{3}, 400 \mathrm{MHz}\right) \delta: 7.09-7.67(\mathrm{~m}, 4 \mathrm{H}$, Ar-H), 3.04 (d, $\left.J=6.2 \mathrm{~Hz}, 2 \mathrm{H}, \mathrm{CH}_{2}\right), 4.18-4.26$ (m, 1H, CH), 9.02 (br s , 1H, P-N-H), 3.91 (d, $J$ $\left.=31.4 \mathrm{~Hz}, 2 \mathrm{H},-\mathrm{OCH}_{2}-\right), 4.58-4.74(\mathrm{~m}, 1 \mathrm{H}, \mathrm{CH}), 3.46\left(\mathrm{q}, 2 \mathrm{H}, \mathrm{OC}_{2} \mathrm{CH}_{3}\right), 1.39(\mathrm{t}, 3 \mathrm{H}$, $\left.\mathrm{OCH}_{2} \underline{\mathrm{C}}_{3}\right), 1.51-1.58\left(\mathrm{~m}, 2 \mathrm{H}, \mathrm{CH}_{2}\right), 1.72-1.79\left(\mathrm{~m}, 2 \mathrm{H}, \mathrm{CH}_{2}\right), 1.09\left(\mathrm{t}, \mathrm{CH}_{3}\right),{ }^{13} \mathrm{C} \mathrm{NMR}\left(\mathrm{CDCl}_{3}\right.$, $100 \mathrm{MHz}$ ) $\delta: 59.7$ (C-3), 45.4 (C-3a), 39.7 (C-4), 131.7 (C-4a), 122.0 (C-5), 124.3 (C-6), 114.1 (C-7), $129.8(\mathrm{C}-8), 142.8(\mathrm{C}-8 \mathrm{a}), 172.3(\mathrm{C}=\mathrm{O}), 51.7\left(\mathrm{OCH}_{2}\right), 54.4(\mathrm{NH}-\mathrm{CH}), 17.6\left(\mathrm{OCH}_{2} \mathrm{CH}_{3}\right)$, $13.3\left(\mathrm{CH}_{2}\right), 13.8\left(\mathrm{CH}_{2}\right), 18.0\left(\mathrm{CH}_{2} \mathrm{CH}_{2} \mathrm{CH}_{3}\right)$; Anal.Calcd. for $\mathrm{C}_{16} \mathrm{H}_{23} \mathrm{~N}_{2} \mathrm{O}{ }_{4} \mathrm{P}: \mathrm{C}, 56.80 ; \mathrm{H}, 6.85 ; \mathrm{N}$, $8.28 \%$ Found: C, 56.75; H, 6.80; N, 8.20\%, $[\alpha]_{\mathrm{D}}(+) 3.1$.

(3a,S)-1-(Proline ethyl ester)-3a,4-dihydro-3H-1 $\lambda^{\mathbf{5}}-[\mathbf{1 , 3}, \mathbf{2}]$ oxazaphospholo[3,4-a] indol-1oxide (5f). Yield 65\%, m.p. 160-163 ${ }^{\circ} \mathrm{C}$. IR (KBr): $v_{\max } 3408(\mathrm{NH}), 1215(\mathrm{P}=\mathrm{O}), 1746(\mathrm{C}=\mathrm{O})$ $\mathrm{cm}^{-1} ;{ }^{31} \mathrm{P} \mathrm{NMR}\left(\mathrm{CDCl}_{3}, 161.9 \mathrm{MHz}\right) \delta: 5.82,{ }^{1} \mathrm{H} \mathrm{NMR}\left(\mathrm{CDCl}_{3}, 400 \mathrm{MHz}\right) \delta: 7.08-7.42(\mathrm{~m}, 4 \mathrm{H}$, Ar-H), 3.19 (d, $\left.J=6.4 \mathrm{~Hz}, 2 \mathrm{H}, \mathrm{CH}_{2}\right), 4.13-4.24(\mathrm{~m}, 1 \mathrm{H}, \mathrm{CH}), 9.58$ (br s , 1H, P-N-H), 3.74 (d, $J$ $\left.=32.4 \mathrm{~Hz}, 2 \mathrm{H},-\mathrm{OCH}_{2}-\right), 3.36\left(\mathrm{q}, 2 \mathrm{H}, \mathrm{OCH}_{2} \mathrm{CH}_{3}\right), 1.17\left(\mathrm{t}, 3 \mathrm{H}, \mathrm{OCH}_{2} \mathrm{CH}_{3}\right), 1.91-2.02(\mathrm{~m}, 2 \mathrm{H}$, $\mathrm{CH}_{2}$ ), 1.64-1.79 (m, 2H, $\left.\mathrm{CH}_{2}\right)$, 2.02-2.21 (t, $2 \mathrm{H}, \mathrm{CH}_{2}$ ); Anal.Calcd. for $\mathrm{C}_{16} \mathrm{H}_{22} \mathrm{~N}_{3} \mathrm{O}_{4} \mathrm{P}: \mathrm{C}, 54.70$; H, 6.31; N, 11.96. Found: C, 54.68; H, 6.29; N, 11.92\%, $[\alpha]_{\mathrm{D}}(+) 3.5$.

(3a, S)-1-(Phenyl alanine ethyl ester)-3a,4-dihydro-3H-1 $\lambda^{5}-[1,3,2]$ oxazaphospholo- $[3,4-a]$ indol-1-oxide (5g). Yield 56\%, m.p. 160-162 ${ }^{\circ} \mathrm{C}$. IR (KBr): $v_{\max } 3442(\mathrm{NH}), 1272(\mathrm{P}=\mathrm{O}), 1736$ $(\mathrm{C}=\mathrm{O}) \mathrm{cm}^{-1},{ }^{31} \mathrm{P} \mathrm{NMR}\left(\mathrm{CDCl}_{3}, 161.9 \mathrm{MHz}\right) \delta: 5.02,{ }^{1} \mathrm{H} \mathrm{NMR}\left(\mathrm{CDCl}_{3}, 400 \mathrm{MHz}\right) \delta: 7.10-7.67$ (m, 9H, Ar-H), 3.03 (d, J=6.4 Hz, 2H, CH ), 4.19-4.29 (m, 1H, CH), 9.86 (br s , 1H, P-N-H), 
$3.65\left(\mathrm{~d}, J=32.2 \mathrm{~Hz}, 2 \mathrm{H},-\mathrm{OCH}_{2}-\right), 4.54-4.68(\mathrm{~m}, 1 \mathrm{H}, \mathrm{CH}), 3.83\left(\mathrm{q}, 2 \mathrm{H}, \mathrm{OC}_{2} \mathrm{CH}_{3}\right), 1.15(\mathrm{t}, 3 \mathrm{H}$, $\left.\mathrm{OCH}_{2} \mathrm{CH}_{3}\right), 1.19\left(\mathrm{~d}, 2 \mathrm{H}, \mathrm{CH}_{2}\right.$ ); Anal. Calcd. for $\mathrm{C}_{20} \mathrm{H}_{23} \mathrm{~N}_{2} \mathrm{O}_{4} \mathrm{P}: \mathrm{C}, 62.17 ; \mathrm{H}, 6.00 ; \mathrm{N}, 7.25 \%$ Found: C, 62.13; H, 5.95; N, 7.20\%, $[\alpha]_{\mathrm{D}}(+) 2.7$.

(3a,S)-1-(Tryptophan ethyl ester)-3a,4-dihydro-3H-1 $\lambda^{5}-[1,3,2]$ oxazaphospholo[3,4-a]indol1-oxide (5h). Yield 69\%, m.p. 146-148 ${ }^{\circ} \mathrm{C}$. IR (KBr): $v_{\max } 3421(\mathrm{NH}), 1226(\mathrm{P}=\mathrm{O}), 1742(\mathrm{C}=\mathrm{O})$ $\mathrm{cm}^{-1} ;{ }^{31} \mathrm{P}$ NMR $\left(\mathrm{CDCl}_{3}, 161.9 \mathrm{MHz}\right) \delta: 5.96,{ }^{1} \mathrm{H} \mathrm{NMR}\left(\mathrm{CDCl}_{3}, 400 \mathrm{MHz}\right) \delta: 6.94-7.67(\mathrm{~m}, 8 \mathrm{H}$, Ar-H), 3.05 (d, $J=6.3 \mathrm{~Hz}, 2 \mathrm{H}, \mathrm{CH}_{2}$ ), 4.12-4.21 (m, 1H, CH), 9.67 (br s , 1H, P-N-H), 10.17 (br s, $1 \mathrm{H}, \operatorname{Ar}-\mathrm{N}-\mathrm{H}), 3.65$ (d, $\left.J=31.9 \mathrm{~Hz}, 2 \mathrm{H},-\mathrm{OCH}_{2}-\right), 3.65\left(\mathrm{q}, 2 \mathrm{H}, \mathrm{OCH}_{2} \mathrm{CH}_{3}\right), 1.17$ (t, $3 \mathrm{H}$, $\mathrm{OCH}_{2} \mathrm{C}_{3}$ ), 3.72-3.86 (m, 1H, CH-C $\left.\underline{H}_{2}\right), 3.91-4.08$ (m, $\left.1 \mathrm{H}, \mathrm{C} \underline{\mathrm{H}}-\mathrm{CH}_{2}\right) ;{ }^{13} \mathrm{C} \mathrm{NMR}\left(\mathrm{CDCl}_{3}, 100\right.$ MHz) $\delta: 52.7$ (C-3), 45.5 (C-3a), 39.7 (C-4), 130.4 (C-4a), 122.1 (C-5), 124.4 (C-6), 113.9 (C-7), 129.6 (C-8), $136.1(\mathrm{C}-8 \mathrm{a}), 169.7(\mathrm{C}=\mathrm{O}), 52.5\left(\mathrm{OCH}_{2}\right), 21.1\left(\mathrm{OCH}_{2} \mathrm{CH}_{3}\right), 56.1(\mathrm{CH}-\mathrm{N}), 39.9$ $\left(\mathrm{NH}-\mathrm{CH}_{2}\right), 39.7\left(\mathrm{NH}-\mathrm{CH}_{2}-\mathrm{CH}\right), 39.2\left(\mathrm{NH}-\mathrm{CH}-\mathrm{CH}_{2}\right), 124.6\left(\mathrm{C}-1^{\prime}\right), 126.4\left(\mathrm{C}-2^{\prime}\right), 131.6\left(\mathrm{C}-3^{\prime}\right)$, 111.5 (C-4'), 136.1 (C-5'), 148.8 (C-6'); Anal.Calcd. for $\mathrm{C}_{22} \mathrm{H}_{27} \mathrm{~N}_{4} \mathrm{O}_{4} \mathrm{P}: \mathrm{C}, 59.72 ; \mathrm{H}, 6.15 ; \mathrm{N}$,

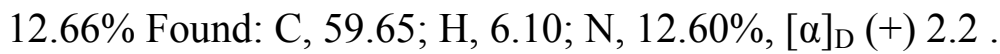

Table 1. Antimicrobial activity of compounds (5a-h) in terms of DIZ in mm

\begin{tabular}{|c|c|c|c|c|c|c|c|c|c|c|c|c|c|c|c|c|}
\hline \multirow[t]{4}{*}{ Compd } & \multicolumn{16}{|c|}{ Zone of inhibition $(\mathrm{mm})$} \\
\hline & \multicolumn{4}{|c|}{$\begin{array}{c}\text { Aspergillus niger } \\
\mu \mathrm{g} / \mathrm{mL}\end{array}$} & \multicolumn{4}{|c|}{$\begin{array}{l}\text { Helminthosporium oryzae } \\
\qquad \mu \mathrm{g} / \mathrm{mL}\end{array}$} & \multicolumn{4}{|c|}{$\begin{array}{c}\text { Escherichia coli } \\
\mu \mathrm{g} / \mathrm{mL}\end{array}$} & \multicolumn{4}{|c|}{$\begin{array}{c}\text { Staphlococcus aureus } \\
\qquad \mu \mathrm{g} / \mathrm{mL} \\
\end{array}$} \\
\hline & 100 & 50 & SD & 25 & 100 & 50 & SD & 25 & 100 & 50 & SD & 2 & 100 & 50 & SD & 25 \\
\hline & & & & & & & & & & & & 5 & & & & \\
\hline $5 \mathrm{a}$ & 8 & 5 & 1.414 & 2 & 11 & 5 & 1.414 & 2 & 10 & 6 & 2.081 & 2 & 9 & 7 & 2.828 & 3 \\
\hline $5 \mathrm{~b}$ & 11 & 8 & 3.535 & 3 & 14 & 9 & 4.760 & 4 & 7 & 8 & 3.535 & 1 & 7 & 6 & 2.081 & 1 \\
\hline $5 \mathrm{c}$ & 10 & 6 & 2.081 & 3 & 12 & 6 & 2.081 & 3 & 13 & 4 & 2.738 & - & 12 & 4 & 2.738 & 2 \\
\hline $5 \mathrm{~d}$ & 12 & 5 & 1.414 & 1 & 10 & 5 & 1.414 & - & 9 & 1 & 1.000 & 2 & 5 & 8 & 3.535 & - \\
\hline $5 \mathrm{e}$ & 10 & 7 & 2.828 & 1 & - & - & 1.414 & - & 7 & 3 & 2.581 & - & 6 & 5 & 1.414 & 2 \\
\hline $5 \mathrm{f}$ & 11 & 7 & 2.828 & 2 & 11 & 5 & 2.828 & 1 & 10 & 5 & 1.414 & 3 & 13 & 5 & 1.414 & 1 \\
\hline $5 \mathrm{~g}$ & 10 & 5 & 1.414 & 1 & 13 & 7 & 3.535 & 2 & 9 & 7 & 2.828 & 2 & 8 & 6 & 2.081 & - \\
\hline $5 \mathrm{~h}$ & 9 & 6 & 2.081 & 3 & 14 & 8 & 2.828 & 1 & 12 & 4 & 2.738 & 1 & 10 & 7 & 2.828 & 1 \\
\hline Bavestin & 15 & 9 & 4.760 & 5 & 16 & 10 & 6.300 & 5 & & & & & & & & \\
\hline $\begin{array}{l}\text { Strepto- } \\
\text { mycin }\end{array}$ & & & & & & & & & 15 & 9 & 4.760 & 5 & 16 & 10 & 6.300 & 5 \\
\hline
\end{tabular}

"-" indicates no activity.

SD Standard deviation. 
Table 2. Minimum inhibitory concentration for $5 \mathrm{a}-\mathrm{h}$ (MIC), $\mu \mathrm{g} / \mathrm{mL}$

\begin{tabular}{ccccc}
\hline Compd. & $\begin{array}{c}\text { Aspergillus } \\
\text { niger }\end{array}$ & $\begin{array}{c}\text { Helminthosporium } \\
\text { oryzae }\end{array}$ & Escherichia coli & $\begin{array}{c}\text { Staphylococcus } \\
\text { aureus }\end{array}$ \\
\hline $\mathbf{5 a}$ & 3.6 & 4.0 & 3.3 & 3.5 \\
$\mathbf{5 b}$ & 4.0 & 3.9 & 4.2 & 3.8 \\
$\mathbf{5 c}$ & 3.9 & 5.3 & 4.1 & 4.0 \\
$\mathbf{5 d}$ & 4.5 & 4.6 & 4.5 & 5.3 \\
$\mathbf{5 e}$ & 3.2 & 3.8 & 3.8 & 3.0 \\
$\mathbf{5 f}$ & 4.7 & 4.2 & 4.0 & 4.5 \\
$\mathbf{5 g}$ & 3.5 & 3.5 & 2.7 & 3.9 \\
$\mathbf{5 h}$ & 4.6 & 3.2 & 3.5 & 4.2 \\
\hline
\end{tabular}

\section{Acknowledgements}

The authors express their thanks to Prof. C. Devendranath Reddy, Dept. of Chemistry, Tirupati, India for encouragement and helpful discussion and the Directors, I.I.Sc. Bangalore and CDRI, Lucknow, India, for the analytical and spectral data.

\section{References}

1. Fest, C.; Schmidt, K. J. The Chemistry of Organophosphorus Pesticides, Springer-Verlag: Berlin, New York, 1982.

2. Szekerke, M. Cancer Treatment Rept. 1976, 60, 374.

3. Cox, P. J. Biochem Pharmacol. 1979, 28, 2045.

4. Dewine, K.G.; Mc Guigan, C.; O’Connor, T. J.; Nicholis, S. R.; Kinchington, D. Aids. 1990, 4, 371 .

5. McGuigan, C.; Narasimhan, P. Síntesis 1993, 311.

6. Rynk, R. Compost Sci. Util. 2000, 8, 1.

7. Kumar, S.; Mukerji, K. G.; Lal, R. Molecular aspects of pesticide degradation by Microorganisms. Crit. Rev. Microbiol. 1996, 22, 1. 
8. (a) Vasu Goverdhan Reddy, P.; Haranath, P.; Suresh Reddy, C.; Naga Raju, C.Indian J. Chem. 2005, 44(B), 1437. (b) Haranath, P.; Anasuyamma, U.; Syam Prasad, G.; Naga Raju, C.; Suresh Reddy, C. Heterocycl. Commun. 2004, 4, 457.

9. Hari Babu, Y.; Vasu Goverdhan Reddy, P.; Suresh Reddy, C.; Devendranath Reddy, C.; Umamaheswari Devi, P. J. Heterocycl. Chem. 2002, 39, 1039.

10. Quin, L. D.; Verkade, J. G. Phosphorus 31 NMR Spectral Properties in Compound Characterization and Structural Analysis, VCH: New York, 1994.

11. Kiran, Y. B.; Gunasekar, D.; Reddy, C. D.; Reddy, C. S.; Tran, K.; Le, T.; Berlin, K. D.; Srinivasan, S.; Devi, M. C. Pest Manage Sci. 2005, 6, 1016.

12. Vincent J. C.; Vincent H. W. Proc. Soc. Expt. Biol. Med. 1944, 55, 162,

13. Benson, H. J., Microbiological Applications, $5^{\text {th }}$ Edn., WC Brown Publications: Boston, 1990.

14. Ita, Z.; Tamas, A. M.; Laszlo, L.; Reijo, S.; Ferenc, F. Tetrahedron 2006, 62, 2883.

15. Webb, R. G.; Haskell, M. W.; Stammer, R. C. J. Org. Chem. 1969, 34, 576.

16. Shahidi Bonjar, G. H, Assin J. Plant Sci. 2004, 3, 56. 